\title{
Registration and Visualization of Transcranial Magnetic Stimulation on MR Images
}

\author{
O. Cuisenaire ${ }^{1}$, M. Ferrant ${ }^{2}$, Y. Vandermeeren ${ }^{3}$, E. Olivier ${ }^{3}$, and B. Macq ${ }^{2}$ \\ 1 Swiss Federal Institute of Technology (EPFL-LTS), Lausanne, Switzerland \\ 2 Université catholique de Louvain (UCL-TELE), Louvain-la-Neuve, Belgium \\ 3 Université catholique de Louvain (UCL-NEFY), Brussels, Belgium \\ Olivier.Cuisenaire@epfl.ch, http://ltswww.epfl.ch/ cuisenai
}

\section{Introduction}

Transcranial Magnetic Stimulation (TMS) has been widely used in the mapping of the primary motor cortex, as well as in the study of language, memory, mood, auditory or visual perception [4. Similarly to EEG or MEG, it provides information - Motor Evoked Potentials (MEP) - located on the surface of the scalp and requires a registration between the physical space $(\mathrm{PhS})$ and a MRI for proper interpretation.

Several methods have been proposed for this registration. Wang [5] and Bastings [1] use a magnetic field (MF) digitizer to acquire points on the scalp surface. Wang attaches the MF transmitter on the patient's head while Basting uses two receivers, one fixed on the patient to track head motion and one mobile to acquire the data. Bastings uses 6 MRI-visible landmarks for registration while Wang uses approx. 400 points for MRI to PhS registration, then landmarks for $\mathrm{PhS}$ to $\mathrm{PhS}$ registration in repeat experiments on the same patient. Ettinger [3] and Potts 4 use a combination of laser to digitize the head surface and optical tracking using LEDs to follow head motion.

In this paper we propose a registration method based on Bastings' and Wang's, but that improves them both in terms of precision and ease of use.

\section{Method}

PhS points are acquired using a MF digitizer (Polhemus Isotrak II). One receiver is fixed on the patient's forehead and the other is a hand held stylus. The scalp surface is characterized by acquiring about 150 points following the pattern illustrated in Figure 11. The coil location and orientation are determined from 3 measures, one at the coil center and the other two in the coil plane.

Both the head (for registration) and the cortical surface (for visualization) are segmented using a semi-automated algorithm based on interactive threshold and mathematical morphology processing. The registration algorithm finds the rigid transform that minimizes the mean square distance between the $\mathrm{PhS}$ digitized points and the MRI head surface. This distance is precomputed using a fast Euclidean distance transform, as explained in details in [2]. 

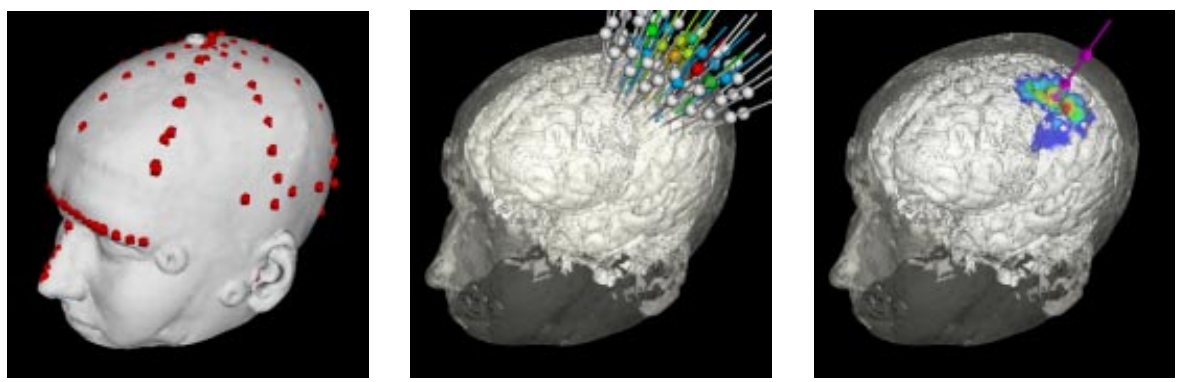

Fig. 1. 1. points used for registration 2. magnet locations 3. interpolated MEPs

Typical results are illustrated at figure 1. The motor cortex was stimulated at points of a regular grid. In Figure 1 2, registered coils centers are represented by spheres and their orientation by lines. The amplitude of the recorded MEP at each location is color coded. In Figure 13 the coil locations are projected onto the brain surface and MEP values are interpolated to form a continuous map. For quantitative analysis, the center of gravity (CoG) of the MEPs is also computed.

\section{Discussion}

The procedure was validated using 4 different methods. Firstly with synthetic $\mathrm{PhS}$ points generated from the MRI scalp to which an arbitrary transform was applied. The algorithm converges for all translations and rotation of up to $30^{\circ}$. The mean residual error was $0.17 \mathrm{~mm}$ over a test set of 100 different arbitrary transforms. Secondly 5 sets of $\mathrm{PhS}$ points were digitized for the same patient and registrations were performed. In average, the residual mean square distance from registered points to scalp surface was $1 \mathrm{~mm}$. Thirdly, those 5 registrations were used to determine the location of several MRI-visible markers. The mean error was found to be $3.8 \mathrm{~mm}$ while the same test using our implementation of Basting's [1] method lead to an error of $9.7 \mathrm{~mm}$. Finally, the reproducibility of the method was assessed by computing the CoGs of the MEPs on the brain surface using the 5 registrations. The average distance to the mean CoG was approx. $1 \mathrm{~mm}, 5$ times less than using Basting's method.

Ease of use was evaluated in terms of operator time required. Scalp digitization requires between 1 and 3 minutes, while each coil location requires approx. 30 seconds. Both the semi-automatic segmentation and the registration take less than a minute. This is orders of magnitude faster than reported by Wang [5].

\section{References}

1. Bastings et al.: Co-registration of cortical magnetic stimulation and functional magnetic resonance imaging. NeuroReport 9 (1998) 1941-1946 
2. Cuisenaire O.: Distance transformation, fast algorithms and applications to medical image processing. Ph.D. Thesis, October 1999, Université catholique de Louvain.

3. Ettinger et al.:Experimentation with a Transscranial Magnetic Stimulation System for Functional Brain Mapping. CVRMed/MRCAS'97, Grenoble, France.

4. Potts G.F. et al.: Visual Hemifield Mapping Using Transcranial Magnetic Stimulation Coregistered with Cortical Surfaces Derived from Magnetic Resonance Images. J Clin Neurophysiology, 15 (1998) 344-350

5. Wang et al.: Head surface digitization and registration: a method for mapping positions on the head onto magnetic resonance images. Brain Topography. 6 (1994) $185-192$ 